\title{
蛇形机器人蜿蜒运动的摩擦机理及推进条件
}

\author{
刘旭鹏 郜志英 藏 勇 张立元 \\ (北京科技大学机械工程学院 北京 100083)
}

\begin{abstract}
摘要: 针对带有从动轮的蛇形机器人, 考虑实际结构中从动轮在蛇形机器人关节连杆上的安装位置, 建立蛇形机器人蜿蜒运 动的动力学模型。提出一种库伦-粘滞混合摩擦力模型, 整体的库伦摩擦力特性和临界点处的粘滞摩擦力特性, 避免了库伦摩 擦力模型本身的非光滑性, 以提高动力学模型的求解效率与计算稳定性。基于改进的动力学与摩擦力模型, 利用摩擦系数比 来表征法向与切向摩擦力的各向异性程度, 通过对蜿蜒运动的仿真分析, 讨论了摩擦系数比对前向运动速度与推进效率的影 响规律, 并对运动过程中蛇形机器人各个关节模块的速度和摩擦力变化进行了分析, 明确了蛇形机器人蜿蜒运动的摩擦学机 理, 解释了蛇的蜿蜒抬起运动能够有效减少摩擦阻力而适用于高速运动的原因。最后通过带从动轮的蛇形机器人样机实验, 对实现蜿蜒运动的摩擦学推进条件进行了验证。研究对于实现蛇形机器人的结构优化, 步态规划与运动控制具有重要的理论 意义与实际指导价值。
\end{abstract}

关键词: 蛇形机器人; 蜿蜒运动; 混合摩擦力; 各向异性; 摩擦机理

中图分类号: TP242

\section{Tribological Mechanism and Propulsion Conditions for Creeping Locomotion of the Snake-like Robot}

\author{
LIU Xupeng GAO Zhiying ZANG Yong ZHANG Liyuan \\ (School of Mechanical Engineering, University of Science and Technology Beijing, Beijing 100083)
}

\begin{abstract}
Considering the installation position of the passive wheel on the joint link of the snake-like robot in the actual structure, the dynamic model of the snake-like robot's creeping locomotion is established. A Coulomb-Viscous mixed friction model is proposed. The overall Coulomb friction characteristics and the Viscous friction characteristics at the critical point can avoid the non smoothness of the Coulomb friction model itself, so as to improve the solution efficiency and computational stability of the dynamic model. Based on the improved dynamics model and friction model, the anisotropic degree of normal friction and tangential friction is represented by the friction coefficient ratio. Through the simulation and analysis of creeping locomotion, the influence of friction coefficient ratio on forward motion speed and propulsion efficiency is discussed, and the velocity and friction variation of each joint module in the moving process of snake-like robot are analyzed. The tribological mechanism of snake-like robot's creeping locomotion explains the reason why the snake's sinus-lifting motion can effectively reduce the friction resistance and is suitable for high-speed motion. Finally, the condition of tribological propulsion to realize the creeping locomotion of the snake-like robot with passive wheels are verified by the prototype experiment. The research in this paper has important theoretical significance and practical guidance value for the realization of snake-like robot structure optimization, gait planning and motion control.
\end{abstract}

Key words: snake-like robot; creeping locomotion; mixed friction; anisotropic; tribological mechanism

\section{0 前言}

蛇是自然界中广泛存在的一种生物, 特殊的无
附肢运动形式使其拥有强大的环境适应能力, 在水 中、草地、沙漠等不同环境中都能灵活运动 ${ }^{[1-3]}$ 。蛇 的运动形式多样, 最常见的包括蜿蜒运动、行波运 动、侧移运动和伸缩运动等 ${ }^{[4]}$, 其中蜿蜒运动是最 典型的运动方式, 具有运动效率高、运动速度快, 运动过程平稳等特点, 而蛇腹部皮肤的各向异性摩

* 中央高校基本科研业务费专项资金资助项目 (FRF-DF-20-13)。 20201019 收到初稿, 20210208 收到修改稿 
擦特性是其实现蜿蜒运动的关键 ${ }^{[5-6]}$ 。

1972 年，日本东京工业大学的 HIROSE 教授研 发了世界上第一个蛇形机器人 ACM-III, 蛇形机器 人全长 $2 \mathrm{~m}$, 质量 $28 \mathrm{~kg}$, 有 20 个相互平行的关节 模块组成, 能够实现平面蜿蜒运动 ${ }^{[7]}$; 之后 HIROSE 教授与其研究团队又相继研发了 ACM-R3、 ACM-R5 等一系列蛇形机器人, 能够在陆地和水中

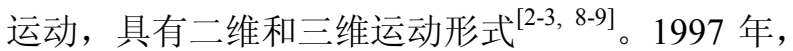
德国的 MILLER 研究团队开始研制蛇形机器人, 至 今已有 S1 至 S7 共七代蛇形机器人, 其中 S5 的不 同关节具有不同的形状尺寸，在外形上具有更强的 仿生效果, S7 则具有距离检测与图像采集功能, 但 S5 和 S7 都只能实现平面二维运动, 无法实现 三维运动 ${ }^{[3,10]}$ 。1999 年, 上海交通大学研发了我 国第一款蛇形机器人样机, 该蛇形机器人总长 $50 \mathrm{~cm}$, 高 $2.6 \mathrm{~cm}$, 宽 $3.5 \mathrm{~cm}$, 由八个关节装置 组成, 在平面内可以做稳定的蜿蜒运动 ${ }^{[11]}$ 。沈阳 自动化研究所也先后研发了巡视者 II、探查者 III 等多种蛇形机器人样机, 并可实现包括蜿蜒运动 的多种运动形式 ${ }^{[1,12-14]}$ 。

与常见的轮式、足式机器人运动方式有所不同, 蛇形机器人的蜿蜒运动依靠关节模块与地面之间的 各向异性摩擦特性实现运动, 因此很大程度上受到 接触地面摩擦状态的影响, 在光滑的地面上运动时 存在严重的打滑现象, 难以向前运动 ${ }^{[15]}$ 。MA 等基 于牛顿-欧拉方程建立了蛇形机器人沿斜面蜿蜒运 动的动力学模型, 并研究了不同斜面倾斜角与运动 波形初始角之间的关系 ${ }^{[16]}$, 进而也讨论了不同摩擦 系数下蛇形机器人的运动速度变化规律, 并指明在 平面上不同摩擦系数比值下与在不同倾斜角的斜面 上具有相似的运动规律 ${ }^{[17]}$ 。LU 等对蛇形机器人在 不同摩擦系数下的运动速度进行了实验研究 ${ }^{[18]}$ 。然 而, 目前对于蛇形机器人实现蜿蜒运动的摩擦学机 理和推进条件尚不明确, 需要运用动力学建模、仿 真分析和样机实验相结合的方法进行进一步的理论 研究和定量分析。

本文针对带从动轮的蛇形机器人, 对实现其蜿 蜒运动的摩擦学机理和推进条件进行了研究。首 先, 考虑实际结构中从动轮在关节连杆上的安装 位置, 建立了更加准确且适用范围更为广泛的动 力学模型; 其次, 提出了一种包含控制参数的 $\tanh$ 函数表示的库伦-粘滞混合摩擦力模型, 克 服了库伦摩擦力模型在速度为零的临界点处的 非光滑特性; 然后, 基于建立的动力学模型和摩 擦力模型, 定义表征摩擦各向异性特征的摩擦系
数比, 讨论了不同摩擦系数比对前向运动速度的 影响规律, 明确了蛇形机器人蜿蜒运动的摩擦学 机理; 最后, 通过蛇形机器人样机实验, 对蛇形 机器人实现蜿蜒运动的摩擦学推进条件进行了 验证。以期为蛇形机器人的结构优化, 步态规划 与运动控制提供参考。

\section{1 蜿蜒运动的动力学模型}

\section{1 基于 Serpenoid 曲线的关节控制模型}

HIROSE 教授基于蛇的运动规律, 最早提出了 Serpenoid 曲线模型, 其曲率方程为 ${ }^{[16,19-21]}$

$$
\kappa\left(s_{0}, s\right)=-\frac{2 K_{n} \pi a_{0}}{L} \sin \left[\frac{2 K_{n} \pi}{L}\left(s_{0}+s\right)\right]+e
$$

式中, $\kappa$ 是 Serpenoid 曲线的曲率, $s_{0}$ 是曲线起始点 距离原点的曲线长度, $S$ 是曲线上的点距离起始点 的曲线长度, $K_{n}$ 是曲线中包含 $S$ 波的个数, $a_{0}$ 是曲 线切线与 $x$ 轴夹角的最大值, 也即波形初始角, $L$ 是 曲线的总长度, $e$ 为曲率偏置量 ${ }^{[22-23]}$ 。

将蛇形机器人等效为由 $N$ 根长度均为 $2 l$ 、质量 分布均匀且首尾相连的连杆组成, 如图 1 所示 ${ }^{[15,24]}$ 。

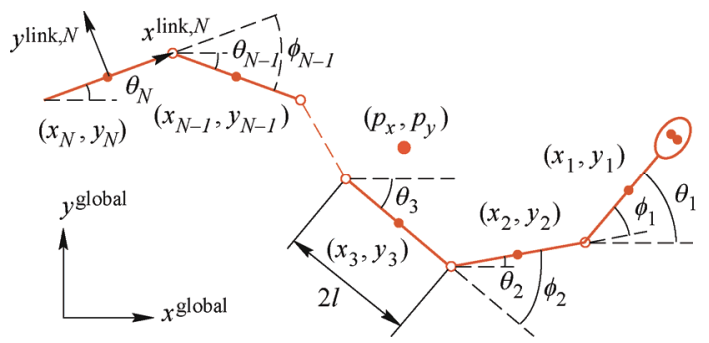

图 1 蛇形机器人连杆模型

定义每根连杆逆时针旋转时为正方向，顺时针 旋转时为负方向, 则蛇形机器人的关节角可表示为

$$
\phi_{i}=\theta_{i}-\theta_{i+1}
$$

式中, $i=1,2, \cdots, N-1$ 。

蛇形机器人的关节角与连杆角之间的关系为

$$
\boldsymbol{\theta}=\boldsymbol{H} \boldsymbol{\phi}
$$

$$
\begin{aligned}
& \boldsymbol{\phi}=\left[\phi_{1}, \phi_{2}, \cdots, \phi_{N-1}, \theta_{N}\right]^{\mathrm{T}} \in \mathbf{R}^{N}, \\
& \boldsymbol{\theta}=\left[\theta_{1}, \theta_{2}, \theta_{3}, \cdots, \theta_{N}\right]^{\mathrm{T}} \in \mathbf{R}^{N}, \\
& \boldsymbol{H}=\left[\begin{array}{cccccc}
1 & 1 & 1 & \cdots & 1 & 1 \\
0 & 1 & 1 & \cdots & 1 & 1 \\
\vdots & \vdots & \vdots & & \vdots & \vdots \\
0 & 0 & 0 & \cdots & 0 & 1
\end{array}\right] \in \mathbf{R}^{N \times N} 。
\end{aligned}
$$

图 1 中所示的蛇形机器人的质心坐标 $\left(p_{x}, p_{y}\right)$ 可表示为 


$$
\left\{\begin{array}{l}
p_{x}=\frac{1}{N m} \sum_{i=1}^{N} m x_{i} \\
p_{y}=\frac{1}{N m} \sum_{i=1}^{N} m y_{i}
\end{array}\right.
$$

式中, $m$ 为蛇形机器人每根关节连杆的质量。

蛇形机器人第 $i$ 和 $i+1$ 节之间的约束条件为

$$
\left\{\begin{array}{l}
x_{i}-x_{i+1}=l \cos \theta_{i}+l \cos \theta_{i+1} \\
y_{i}-y_{i+1}=l \sin \theta_{i}+l \sin \theta_{i+1}
\end{array}\right.
$$

式中, $i=1,2, \cdots, N-1$ 。

根据曲率定义

$$
\kappa=\lim _{\Delta s \rightarrow 0} \frac{\Delta \alpha}{\Delta s}=\frac{\mathrm{d} \alpha}{\mathrm{d} s}
$$

式中, $\Delta s$ 表示弧长长度, $\Delta \alpha$ 表示弧长两端点的切 线之间的夹角。

根据图 1 与式(6), 蛇形机器人的关节角 $\phi_{i}$ 可表 示为

$$
\phi_{i}=\Delta \alpha_{i}=\int_{a}^{b} \kappa \mathrm{d} s
$$

式中, $a 、 b$ 分别表示前后相邻连杆质心位置与坐标 原点之间的曲线长度。

将式(1)代入式(7)得到蛇形机器人离散化后的 关节角

$$
\begin{aligned}
\phi_{i}= & \int_{s_{0}+2(N-i-1) l+l}^{s_{0}+2(N-i) l+l} \kappa(u) \mathrm{d} u= \\
& \alpha \sin \left[\beta s_{0}+(N-i) \delta\right]+\varphi_{0}
\end{aligned}
$$

式中, $\alpha=-2 a_{0} \sin \left(\frac{K_{n} \pi}{N}\right), \beta=\frac{2 K_{n} \pi}{L}, \delta=\frac{2 K_{n} \pi}{N}$, $\varphi_{0}=2 e l$ 。

将式(8)转换为时间 $t$ 的函数为

$$
\phi_{i}(t)=\alpha \sin [\omega t+(N-i) \delta]+\varphi_{0}
$$

式中, $\omega=\frac{2 K_{n} \pi}{T}, T$ 为蛇形机器人的关节变化 $K_{n}$ 个 周期所用的时间 ${ }^{[19]}$ 。

\section{2 考虑从动轮位置的动力学建模}

在蛇形机器人实际的结构中, 从动轮的位置可 能没有在连杆模块的中心处, 这使得连杆所受摩擦 力的作用位置与连杆中心位置具有一定的偏置距 离。在 PETTERSEN 等关于蛇形机器人平面蜿蜒运 动的动力学模型中将摩擦力的作用点位置简化到连 杆的中心 ${ }^{[25-27]}$ 。为了使动力学模型与实际情况更加 符合, 也便于分析从动轮的位置对蛇形机器人蜿蜒 运动的影响规律, 本文考虑从动轮在关节连杆上的 安装位置, 也即连杆在运动过程中所受摩擦力作用 点在连杆上的位置, 对蛇形机器人蜿蜒运动的动力
学模型进行了改进。

考虑摩擦力作用点在关节连杆上的位置后, 蛇形 机器人第 $i$ 节关节连杆的受力情况如图 2 所示 ${ }^{[15,28]}$ 。

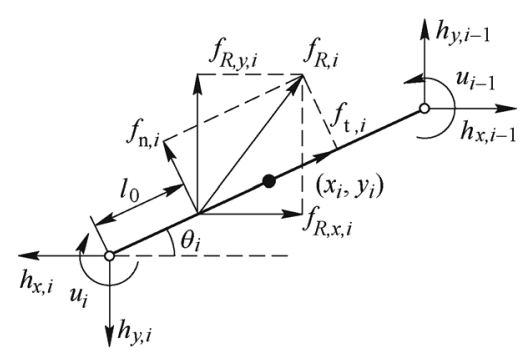

图 2 蛇形机器人第 $i$ 节关节模块

根据图 2, 由刚体的平面运动定理得到第 $i$ 个关 节连杆的动力学方程

$$
\left\{\begin{array}{l}
m \ddot{x}_{i}=f_{R, x, i}+h_{x, i-1}-h_{x, i} \\
m \ddot{y}_{i}=f_{R, y, i}+h_{y, i-1}-h_{y, i} \\
J \ddot{\theta}_{i}=u_{i-1}-u_{i}-l \sin \theta_{i}\left(h_{x, i}+h_{x, i-1}\right)+ \\
l \cos \theta_{i}\left(h_{y, i}+h_{y, i-1}\right)+\left(l-l_{0}\right) \sin \theta_{i} f_{R, x, i}- \\
\left(l-l_{0}\right) \cos \theta_{i} f_{R, y, i}
\end{array}\right.
$$

式中, $i=1,2, \cdots, N, h_{x, 0}=0, h_{x, N}=0, h_{y, 0}=0$, $h_{y, N}=0, u_{0}=0, u_{N}=0$ 。

根据式(4)、(5)、(10)得到

$$
\left\{\begin{array}{l}
\mathbf{D}^{\mathrm{T}} \boldsymbol{u}=\boldsymbol{V}_{1} \ddot{\boldsymbol{\theta}}+\boldsymbol{V}_{2} \dot{\boldsymbol{\theta}}^{2}+\boldsymbol{V}_{3} \boldsymbol{f}_{R, x}+\boldsymbol{V}_{4} \boldsymbol{f}_{R, y} \\
N m \ddot{\boldsymbol{P}}=N m\left[\begin{array}{l}
\ddot{P}_{x} \\
\ddot{P}_{y}
\end{array}\right]=\left[\begin{array}{l}
\boldsymbol{e}^{\mathrm{T}} \boldsymbol{f}_{R, x} \\
\boldsymbol{e}^{\mathrm{T}} \boldsymbol{f}_{R, y}
\end{array}\right]
\end{array}\right.
$$

$$
\begin{aligned}
& \boldsymbol{V}_{1}=-J \boldsymbol{I}_{N}-m l^{2}\left[\boldsymbol{S}_{\theta} \boldsymbol{K} \boldsymbol{S}_{\theta}+\boldsymbol{C}_{\theta} \boldsymbol{K} \boldsymbol{C}_{\theta}\right] \\
& \boldsymbol{V}_{2}=-m l^{2}\left[\boldsymbol{S}_{\theta} \boldsymbol{K} \boldsymbol{C}_{\theta}-\boldsymbol{C}_{\theta} \boldsymbol{K} \boldsymbol{S}_{\theta}\right] \\
& \boldsymbol{V}_{3}=-l \boldsymbol{S}_{\theta} \boldsymbol{A}^{\mathrm{T}}\left(\boldsymbol{D} \boldsymbol{D}^{\mathrm{T}}\right)^{-1} \boldsymbol{D}+\left(l-l_{0}\right) \boldsymbol{S}_{\theta} \\
& \boldsymbol{V}_{4}=l \boldsymbol{C}_{\theta} \boldsymbol{A}^{\mathrm{T}}\left(\boldsymbol{D} \boldsymbol{D}^{\mathrm{T}}\right)^{-1} \boldsymbol{D}-\left(l-l_{0}\right) \boldsymbol{C}_{\theta} \\
& \boldsymbol{K}=\boldsymbol{A}^{\mathrm{T}}\left(\boldsymbol{D} \boldsymbol{D}^{\mathrm{T}}\right)^{-1} \boldsymbol{A}
\end{aligned}
$$

$$
\begin{aligned}
& \boldsymbol{A}=\left[\begin{array}{ccccc}
1 & 1 & & & \\
& 1 & 1 & & \\
& & \ddots & \ddots & \\
& & & 1 & 1
\end{array}\right] \in \mathbf{R}^{(N-1) \times N} \\
& \boldsymbol{D}=\left[\begin{array}{ccccc}
1 & -1 & & & \\
& 1 & -1 & & \\
& & \ddots & \ddots & \\
& & & 1 & -1
\end{array}\right] \in \mathbf{R}^{(N-1) \times N} \\
& \boldsymbol{S}_{\theta}=\operatorname{diag}(\sin \boldsymbol{\theta}) \in \mathbf{R}^{N \times N} \\
& \boldsymbol{C}_{\theta}=\operatorname{diag}(\cos \boldsymbol{\theta}) \in \mathbf{R}^{N \times N} \\
& \sin \boldsymbol{\theta}=\left[\sin \theta_{1}, \sin \theta_{2}, \cdots, \sin \theta_{N}\right]^{\mathrm{T}} \in \mathbf{R}^{N}
\end{aligned}
$$


$\cos \boldsymbol{\theta}=\left[\cos \theta_{1}, \cos \theta_{2}, \cdots, \cos \theta_{N}\right]^{\mathrm{T}} \in \mathbf{R}^{N}$

$$
\begin{gathered}
\boldsymbol{I}_{N}=\left[\begin{array}{cccc}
1 & 0 & \cdots & 0 \\
0 & 1 & \cdots & 0 \\
\vdots & \vdots & & \vdots \\
0 & 0 & \cdots & 1
\end{array}\right] \in \mathbf{R}^{N \times N} \\
\boldsymbol{f}_{R, x}=\left[f_{R, x, 1}, f_{R, x, 2}, \cdots, f_{R, x, N}\right]^{\mathrm{T}} \in \mathbf{R}^{N} \\
\boldsymbol{f}_{R, y}=\left[f_{R, y, 1}, f_{R, y, 2}, \cdots, f_{R, y, N}\right]^{\mathrm{T}} \in \mathbf{R}^{N}
\end{gathered}
$$

对于由 $N$ 根连杆组成的蛇形机器人, 包含 $N-1$ 关节。蛇形机器人具有 $N+2$ 个自由度, 其中 $N-1$ 个自由度是可控的驱动自由度, 为蛇形机器人 的 $N-1$ 个关节的控制输入, 其余 3 个自由度是不可 控的圥余自由度, 分别为最后一根连杆的绝对角度 $\theta_{N}$ 和蛇形机器人整体的质心位置坐标 $p_{x} 、 p_{y}$ 。为 了使动力学模型转化为便于求解的形式, 利用式(3) 将式(11)中的输入自由度和午余自由度分离后得到

$$
\left\{\begin{array}{l}
\boldsymbol{M}_{11} \ddot{\boldsymbol{q}}_{a}+\boldsymbol{M}_{12} \ddot{\boldsymbol{q}}_{u}+\boldsymbol{W}_{1}+\boldsymbol{G}_{1} \boldsymbol{f}_{R}=\boldsymbol{u} \\
\boldsymbol{M}_{21} \ddot{\boldsymbol{q}}_{a}+\boldsymbol{M}_{22} \ddot{\boldsymbol{q}}_{u}+\boldsymbol{W}_{2}+\boldsymbol{G}_{2} \boldsymbol{f}_{R}=\boldsymbol{0}_{3 \times 1}
\end{array}\right.
$$

式中 $\boldsymbol{q}_{a}=\left[\phi_{1}, \phi_{2}, \cdots, \phi_{N-1}\right]^{\mathrm{T}} \in \mathbf{R}^{N-1}$ 表示驱动自由度;

$$
\begin{aligned}
& \boldsymbol{q}_{u}=\left[\theta_{N}, p_{x}, p_{y}\right]^{\mathrm{T}} \in \mathbf{R}^{3} \text { 表示圥余自由度; } \\
& \boldsymbol{M}_{11} \in \mathbf{R}^{(N-1) \times(N-1)}, \boldsymbol{M}_{12} \in \mathbf{R}^{(N-1) \times 3} ; \\
& \boldsymbol{M}_{21} \in \mathbf{R}^{3 \times(N-1)}, \boldsymbol{M}_{22} \in \mathbf{R}^{3 \times 3} ; \\
& \boldsymbol{W}_{1} \in \mathbf{R}^{N-1}, \boldsymbol{W}_{2} \in \mathbf{R}^{3} ; \\
& \boldsymbol{G}_{1} \in \mathbf{R}^{(N-1) \times 2 N}, \boldsymbol{G}_{2} \in \mathbf{R}^{3 \times 2 N} ; \\
& \boldsymbol{f}_{R}=\left[\boldsymbol{f}_{R, x}, \boldsymbol{f}_{R, y}\right]^{\mathrm{T}} \in \mathbf{R}^{2 N} \text { 。 }
\end{aligned}
$$

引入一个指数稳定关节控制器 $\overline{\boldsymbol{u}}, \overline{\boldsymbol{u}}$ 表示蛇形 机器人关节的控制输入

$$
\overline{\boldsymbol{u}}=\ddot{\boldsymbol{\phi}}_{\mathrm{ef}}+k_{\mathrm{d}}\left(\dot{\phi}_{\mathrm{ref}}-\dot{\boldsymbol{\phi}}\right)+k_{\mathrm{p}}\left(\phi_{\mathrm{eff}}-\boldsymbol{\phi}\right)
$$

式中, $\phi_{\text {ref }}=\left[\phi_{1, \text { ref }}, \cdots, \phi_{N-1, \text { ref }}\right]^{\mathrm{T}} \in \mathbf{R}^{N-1}$, 表示蛇形机 器人关节角的参考角度, 采用式(9)的关节控制函 数, 有

$$
\left\{\begin{array}{l}
\phi_{\mathrm{ref}, i}=\alpha \sin (\omega t+(N-i) \delta)+\varphi_{0} \\
\dot{\phi}_{\mathrm{ref}, i}=\alpha \omega \cos (\omega t+(N-i) \delta) \\
\ddot{\phi}_{\mathrm{ref}, i}=-\alpha \omega^{2} \sin (\omega t+(N-i) \delta)
\end{array}\right.
$$

令 $\ddot{\boldsymbol{q}}_{a}=\overline{\boldsymbol{u}}$, 代入式(12)中的第二个方程, 得到 蛇形机器人的咒余自由度 $\ddot{\boldsymbol{q}}_{u}$ 为

$$
\ddot{\boldsymbol{q}}_{u}=-\boldsymbol{M}_{22}^{-1}\left(\boldsymbol{W}_{2}+\boldsymbol{G}_{2} \boldsymbol{f}_{R}\right)-\boldsymbol{M}_{22}^{-1} \boldsymbol{M}_{21} \overline{\boldsymbol{u}}
$$

将式(15)代入式(12)的第一个方程, 得到

$$
\begin{gathered}
\boldsymbol{u}=\left(\boldsymbol{M}_{11}-\boldsymbol{M}_{12} \boldsymbol{M}_{22}^{-1} \boldsymbol{M}_{21}\right) \overline{\boldsymbol{u}}-\boldsymbol{M}_{12} \boldsymbol{M}_{22}^{-1} \boldsymbol{W}_{2}- \\
\boldsymbol{M}_{12} \boldsymbol{M}_{22}^{-1} \boldsymbol{G}_{2} \boldsymbol{f}_{R}+\boldsymbol{W}_{1}+\boldsymbol{G}_{1} \boldsymbol{f}_{R}
\end{gathered}
$$

根据式(16), 可以得到蛇形机器人内部的关节 力矩, 为了简化动力学模型以便于方程的求解, 本 文不考虑关节力矩 $\boldsymbol{u}$, 结合式(15)得到蛇形机器人 的动力学方程

$$
\left\{\begin{array}{l}
\ddot{\boldsymbol{q}}_{a}=\overline{\boldsymbol{u}} \\
\ddot{\boldsymbol{q}}_{u}=-\boldsymbol{M}_{22}^{-1}\left(\boldsymbol{W}_{2}+\boldsymbol{G}_{2} \boldsymbol{f}_{R}\right)-\boldsymbol{M}_{22}^{-1} \boldsymbol{M}_{21} \overline{\boldsymbol{u}}
\end{array}\right.
$$

定义 $\boldsymbol{x}_{1}=\boldsymbol{q}_{a}, \boldsymbol{x}_{2}=\dot{\boldsymbol{q}}_{a}, \boldsymbol{x}_{3}=\boldsymbol{q}_{u}, \boldsymbol{x}_{4}=\dot{\boldsymbol{q}}_{u}$, 将 式(17)的动力学模型转化为微分方程组的形式为

$$
\dot{\boldsymbol{x}}=\left[\begin{array}{c}
\dot{\boldsymbol{x}}_{1} \\
\dot{\boldsymbol{x}}_{2} \\
\dot{\boldsymbol{x}}_{3} \\
\dot{\boldsymbol{x}}_{4}
\end{array}\right]=\left[\begin{array}{c}
\boldsymbol{x}_{2} \\
\overline{\boldsymbol{u}} \\
\boldsymbol{x}_{4} \\
\ddot{\boldsymbol{q}}_{u}
\end{array}\right]
$$

\section{2 混合摩擦力模型及推进条件}

蛇形机器人在平面蜿蜒运动过程中, 关节连杆 与地面之间的摩擦力为蛇形机器人提供前行方向上 的推进力, 通常有库伦摩擦力和粘滞摩擦力两种模 型。库伦摩擦力模型用于描述蛇形机器人在普通平 地上的运动情况, 粘滞摩擦力模型用于描述蛇形机 器人在水中的运动情况。在实际工程中, 通常在蛇 形机器人的腹部安装从动轮以实现非完整约束。

\section{1 带有从动轮的各向异性摩擦力}

从动轮和地面之间的摩擦力为各向同性的库伦 摩擦力, 假设沿垂直于从动轮转动轴的方向为纯滚 动, 从动轮和转动轴间存在一个力矩 $\tau, \tau$ 的方向 与从动轮转动方向相反, 则从动轮的受力情况如 图 3 所示。

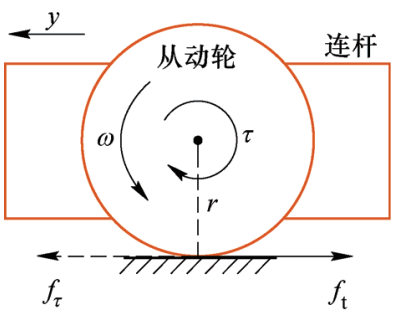

图 3 从动轮受力分析

若从动轮的半径为 $r$, 则力矩 $\tau$ 转换到接触点 处的等效力 $f_{\tau}$ 为

$$
f_{\tau}=\tau / r
$$

等效力 $f_{\tau}$ 与摩擦力 $f_{\mathrm{t}}$ 平衡, 即

$$
f_{\tau}=f_{\mathrm{t}}
$$

式中, $f_{\mathrm{t}}$ 为关节连杆所受的切向摩擦力。

根据库伦摩擦力模型, 切向摩擦力 $f_{\mathrm{t}}$ 的方向与 
从动轮转动轴的方向垂直, 其大小可表示为

$$
f_{\mathrm{t}}=\mu_{\mathrm{t}} m g
$$

法向摩擦力 $f_{\mathrm{n}}$ 的方向与从动轮转动轴的方向 平行, 其大小可表示为

$$
f_{\mathrm{n}}=\mu_{\mathrm{n}} m g
$$

因此, 根据式(19) $(21)$, 切向摩擦系数 $\mu_{\mathrm{t}}$ 为

$$
\mu_{\mathrm{t}}=\frac{\tau}{m g r}
$$

根据式(23), 切向摩擦系数 $\mu_{\mathrm{t}}$ 为等效的摩擦系 数, 主要由从动轮和转动轴间的摩擦力矩决定, 通 过改变摩擦力矩大小, 就能改变等效后的切向摩擦 系数。法向摩擦系数 $\mu_{\mathrm{n}}$ 为从动轮和地面之间的动摩 擦系数, 主要由从动轮和地面的材料、接触表面性 质等因素决定, 当从动轮的材料、表面性质等因素 保持不变时, 在不同材料、表面性质的地面上运动 时, 从动轮和地面之间具有不同的法向摩擦系数。 因此, 虽然从动轮和地面之间是各向同性的库伦摩 擦力, 但关节连杆则受到各向异性摩擦力。

\section{2 库伦-粘滞混合摩擦力模型}

由于库伦摩擦力模型在速度为零的临界点处存 在非光滑性, 因此基于库伦摩擦力模型的蛇形机器 人动力学模型难以实现数值求解 ${ }^{[29]}$ 。为了解决这一 问题, 本文提出一种库伦-粘滞混合摩擦力模型, 该 混合摩擦力模型将库伦摩擦力模型的非光滑部分用 粘滞摩擦力模型代替, 而其他部分采用库伦摩擦力 模型, 最终呈现出全局的库伦摩擦力特性和临界点 处的局部粘滞摩擦力特性。

摩擦力模型用含参数 $\lambda$ 的 $\tanh$ 函数表征, 即

$$
f(v)=\tanh (\lambda v)
$$

式中, $\lambda$ 主要决定函数曲线的形状; $v$ 表示连杆的 切向方向速度或法向方向速度, 不同 $\lambda$ 值时的速度 与函数值的关系如图 4 所示。

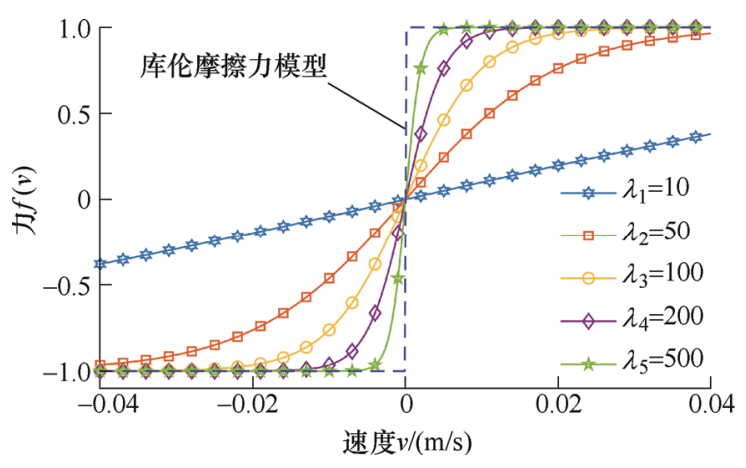

图 4 不同 $\lambda$ 时的函数值

根据图 4 可知, 当取不同的 $\lambda$ 值时, 曲线具有
不同的形状, $\lambda$ 值越大时曲线在 0 附近的斜率越大, 越接近非光滑状态的库伦摩擦力模型, 本文取 $\lambda=200$ 。

库伦-粘滞混合摩擦力模型主要包含了两部分 摩擦力特性: 一部分是在 $v=0$ 的局部范围内呈现出 的粘滞摩擦力特性; 另一部分是全局范围内呈现出 的库伦摩擦力特性, 如图 5 所示。

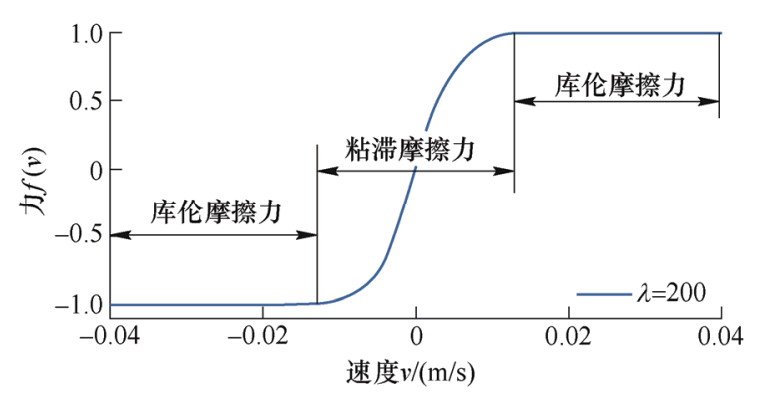

图 5 混合摩擦力模型

库伦一粘滞混合摩擦力模型是全局光滑的, 在 数学上避免了库伦摩擦力函数本身的非光滑性, 以 提高动力学模型的求解效率与计算稳定性。根据库 伦-粘滞混合摩擦力模型, 蛇形机器人单个关节模块 在全局坐标系中的摩擦力可表示为

$$
f_{R, i}^{\text {global }}=-m g \boldsymbol{R}\left[\begin{array}{cc}
\mu_{\mathrm{t}} & 0 \\
0 & \mu_{\mathrm{n}}
\end{array}\right] \tanh \left(\lambda\left[\begin{array}{c}
v_{\mathrm{t}, i} \\
v_{\mathrm{n}, i}
\end{array}\right]\right)
$$

式中, $\boldsymbol{R}=\left[\begin{array}{cc}\cos \theta_{i} & -\sin \theta_{i} \\ \sin \theta_{i} & \cos \theta_{i}\end{array}\right]$, 为局部坐标系到全局 坐标系的变换矩阵, $\mu_{\mathrm{t}} 、 \mu_{\mathrm{n}}$ 分别为切向摩擦系数 和法向摩擦系数。

蛇形机器人所有关节模块在全局坐标系中的摩 擦力为

$$
\begin{gathered}
\boldsymbol{f}_{R}=-m g \boldsymbol{R}_{1} \tanh \left(\lambda\left[\begin{array}{c}
\boldsymbol{v}_{\mathrm{t}} \\
\boldsymbol{v}_{\mathrm{n}}
\end{array}\right]\right) \\
{\left[\begin{array}{c}
\boldsymbol{v}_{\mathrm{t}} \\
\boldsymbol{v}_{\mathrm{n}}
\end{array}\right]=\left[\begin{array}{c}
\mathbf{0}_{N \times 1} \\
\left(l_{0}-l\right) \dot{\boldsymbol{\theta}}
\end{array}\right]+\boldsymbol{R}_{2}\left[\begin{array}{c}
\dot{\boldsymbol{X}} \\
\dot{\boldsymbol{Y}}
\end{array}\right]} \\
\boldsymbol{R}_{1}=\left[\begin{array}{cc}
\mu_{\mathrm{t}} \boldsymbol{C}_{\theta} & -\mu_{\mathrm{n}} \boldsymbol{S}_{\theta} \\
\mu_{\mathrm{t}} \boldsymbol{S}_{\theta} & \mu_{\mathrm{n}} \boldsymbol{C}_{\theta}
\end{array}\right] \in \mathbf{R}^{2 N \times 2 N} \\
\boldsymbol{R}_{2}=\left[\begin{array}{cc}
\boldsymbol{C}_{\theta} & \boldsymbol{S}_{\theta} \\
-\boldsymbol{S}_{\theta} & \boldsymbol{C}_{\theta}
\end{array}\right] \in \mathbf{R}^{2 N \times 2 N}
\end{gathered}
$$

$\left(l_{0}-l\right) \dot{\boldsymbol{\theta}}$ 表示连杆质心位置的速度转化到摩擦 力作用点处的法向方向上的速度增量。

\section{3 蜿蜒运动的摩擦推进条件}

蛇形机器人蜿蜒运动过程中的推进力由摩擦力 产生, 当摩擦力方向与蛇形机器人的前进方向一致 
时起到了推动蛇形机器人运动的作用, 反之则成为 蛇形机器人的运动阻力。

为了得到蛇形机器人各个关节模块的摩擦力, 根据式(25) (27), 得到蛇形机器人各个关节模块在 切向和法向方向上的摩擦力为

$$
\begin{gathered}
f_{R, \mathrm{t}, i}=-m g \mu_{\mathrm{t}} \cos \theta_{i} \tanh \left(\zeta_{1}\right) \\
f_{R, \mathrm{n}, i}=-m g \mu_{\mathrm{n}} \cos \theta_{i} \tanh \left(\zeta_{2}\right) \\
\zeta_{1}=\lambda \cos \theta_{i} \dot{x}_{i}+\lambda \sin \theta_{i} \dot{y}_{i} \\
\zeta_{2}=-\lambda \sin \theta_{i} \dot{x}_{i}+\lambda \cos \theta_{i} \dot{y}_{i}+\lambda\left(l_{0}-l\right) \cdot \dot{\theta}_{i}
\end{gathered}
$$

在 $x 、 y$ 方向上的摩擦力为

$$
\begin{aligned}
f_{R, x, i}= & -m g \mu_{\mathrm{t}} \cos \theta_{i} \tanh \left(\zeta_{1}\right)+ \\
& m g \mu_{\mathrm{n}} \sin \theta_{i} \tanh \left(\zeta_{2}\right) \\
f_{R, y, i}= & -m g \mu_{\mathrm{t}} \sin \theta_{i} \tanh \left(\zeta_{1}\right)- \\
& m g \mu_{\mathrm{n}} \cos \theta_{i} \tanh \left(\zeta_{2}\right)
\end{aligned}
$$

蛇形机器人各个关节摩擦力在 $x 、 y$ 方向上的 摩擦力合力为

$$
\begin{aligned}
f_{R, x}= & -\sum_{i=1}^{N}\left[m g \mu_{\mathrm{t}} \cos \theta_{i} \tanh \left(\zeta_{1}\right)-\right. \\
& \left.m g \mu_{\mathrm{n}} \sin \theta_{i} \tanh \left(\zeta_{2}\right)\right] \\
f_{R, y}= & -\sum_{i=1}^{N}\left[m g \mu_{\mathrm{t}} \sin \theta_{i} \tanh \left(\zeta_{1}\right)+\right. \\
& \left.m g \mu_{\mathrm{n}} \cos \theta_{i} \tanh \left(\zeta_{2}\right)\right]
\end{aligned}
$$

式中, $f_{R, x}$ 表示蛇形机器人在运动过程中的推进力 合力, 当 $f_{R, x}>0$ 时起到运动推进力的作用, 当 $f_{R, x}<0$ 时则起到运动阻力的作用。

\section{3 不同摩擦条件下的蜿蜒运动仿真}

蛇形机器人的平面蜿蜒在不同摩擦系数条件下 伴有不同程度的滑移现象, 这种滑移也导致了蛇形 机器人前向运动速度大小和方向难以准确预测和控 制, 甚至在极为苛刻的条件下蛇形机器人根本无法 实现蜿蜒运动。蛇形机器人各个关节模块受到的摩 擦力以及运动速度的变化规律不仅能够反映蛇形机 器人的蜿蜒运动机理, 还能够体现出在运动过程中 各个关节模块的运动状态和滑移程度。

\section{1 各个关节模块的摩擦力及运动速度}

蛇形机器人的蜿蜒运动中, 波形的传递方向与 蛇形机器人的运动方向相反, 沿着蛇形机器人的前 进方向, 定义左侧幅值最大处为波峰, 右侧幅值最 大处为波谷, 在对称于波峰和波谷的中轴线位置处,
由波谷变化到波峰的拐点为上拐点, 由波峰变化到 波谷的拐点为下拐点, 如图 6 所示。

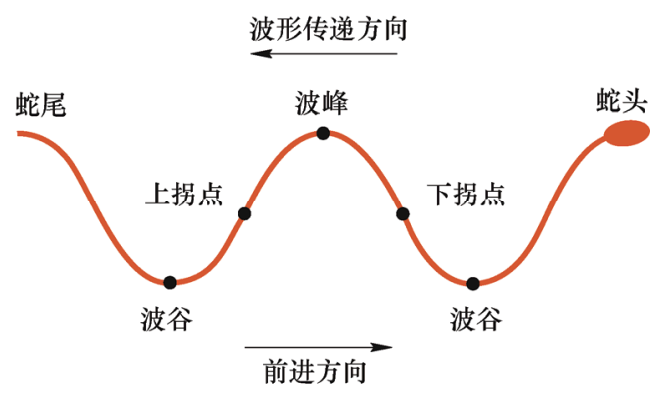

图 6 生物蛇的运动状态示意图

根据式(4)、(5)得到蛇形机器人各个关节模块的 质心位置及运动速度为

$$
\begin{aligned}
& \left\{\begin{array}{l}
\boldsymbol{X}=\boldsymbol{T}^{-1}\left[\begin{array}{c}
l \boldsymbol{A} \cos \boldsymbol{\theta} \\
p_{x}
\end{array}\right] \\
\boldsymbol{Y}=\boldsymbol{T}^{-1}\left[\begin{array}{c}
l \boldsymbol{A} \sin \boldsymbol{\theta} \\
p_{y}
\end{array}\right]
\end{array}\right. \\
& \left\{\begin{array}{l}
\dot{\boldsymbol{X}}=\boldsymbol{T}^{-1}\left[\begin{array}{c}
-l \boldsymbol{A} \boldsymbol{S}_{\theta} \dot{\boldsymbol{\theta}} \\
\dot{p}_{x}
\end{array}\right] \\
\dot{\boldsymbol{Y}}=\boldsymbol{T}^{-1}\left[\begin{array}{c}
l \boldsymbol{A} \boldsymbol{C}_{\theta} \dot{\boldsymbol{\theta}} \\
\dot{p}_{y}
\end{array}\right]
\end{array}\right.
\end{aligned}
$$

根据式(30)、(31)能够得到蛇形机器人每个关节 模块在运动过程中 $x 、 y$ 方向上的摩擦力, 根据式 (35)能够得到蛇形机器人各个关节模块的质心在 $x$ 、 $y$ 方向上的速度，通过坐标变换得到质心在切向和 法向方向上的运动速度。设置仿真参数如表 1 所示。

表 1 摩擦力和速度的参数取值

\begin{tabular}{cc}
\hline 参数 & 数值 \\
\hline 切向摩擦系数 $\mu_{\mathrm{t}}$ & 0.05 \\
法向摩擦系数 $\mu_{\mathrm{n}}$ & 0.2 \\
波形初始角 $\left.a_{0} /{ }^{(}\right)$ & 40 \\
波形个数 $K_{n}$ & 2 \\
关节连杆数量 $N$ & 11 \\
关节连杆长度一半 $l / \mathrm{m}$ & 0.1059 \\
从动轮位置 $l_{0} / \mathrm{m}$ & 0.1059 \\
周期 $T / \mathrm{s}$ & 10 \\
关节连杆质量 $m / \mathrm{kg}$ & 0.268 \\
关节连杆转动惯量 $J /\left(\mathrm{kg} \cdot \mathrm{m}^{2}\right)$ & 0.001 \\
\hline
\end{tabular}

利用 Matlab 进行数值仿真, 得到蛇形机器人各 个关节模块的摩擦力和运动速度。由于蛇形机器人 各个关节模块的摩擦力和运动速度的变化规律关于 
蛇形机器人中心位置的关节模块前后近似对称, 如 第一个关节模块和最后一个关节模块的变化规律相 似, 随蛇形机器人的关节模块绝对角 $\theta_{i}$ 呈周期性变 化, 并形成一条闭合曲线, 因此, 针对前六个关节 模块进行分析。各个关节模块的摩擦力和速度如图

7、图 8 所示。
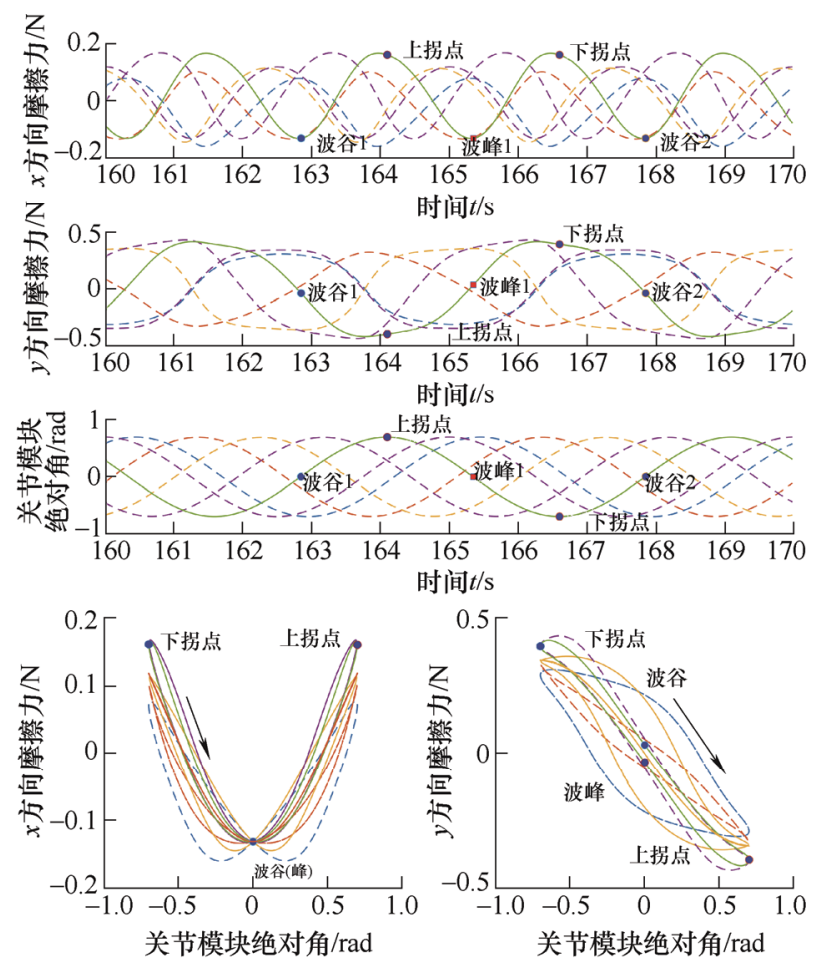

图 7 关节模块 1 6 的 $x 、 y$ 方向摩擦力
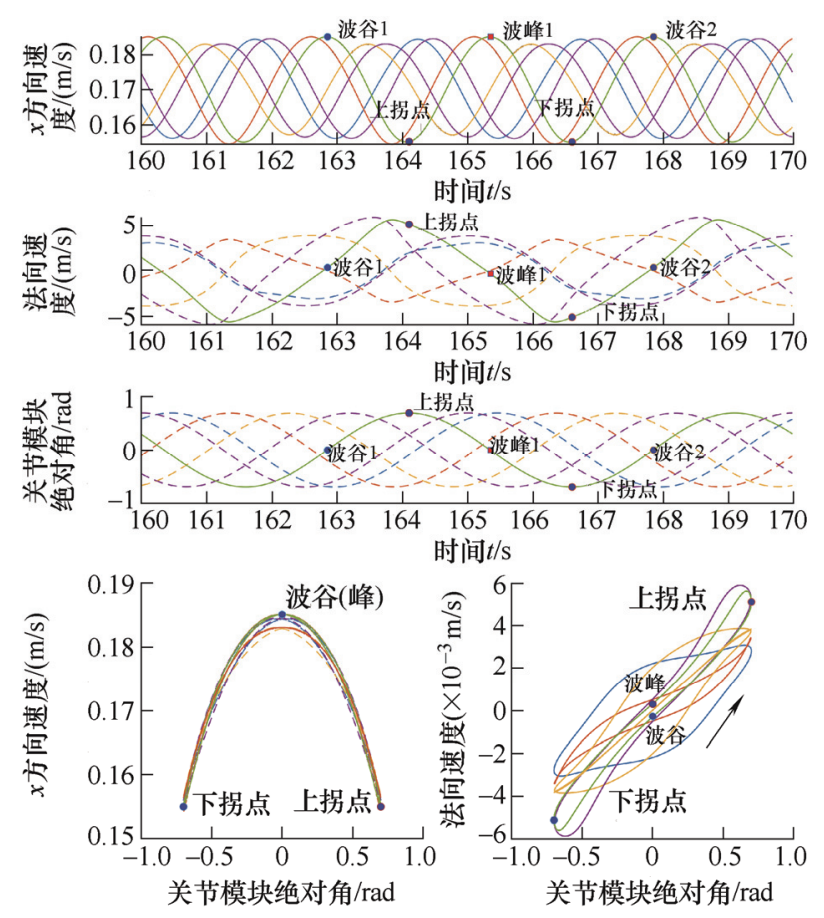

图 8 关节模块 1 6 的 $x$ 方向、法向方向速度
图 7 中, 前三个子图为蛇形机器人前六个关节 模块的 $x 、 y$ 方向摩擦力以及绝对角随时间的变化 关系，后两个子图为 $x 、 y$ 方向摩擦力与绝对角的 相图。针对第五个关节模块，当其处于上拐点或下 拐点位置附近, 即 $\theta_{i}= \pm a_{0}$ 附近时, 其 $x 、 y$ 方向上 的摩擦力达到了最大值, 此时 $x$ 方向上的摩擦力与 前进方向一致, 起到了运动推进力的作用; 当其处 于波峰或波谷位置附近, 即 $\theta_{i}=0$ 附近时, $x$ 方向上 的摩擦力达到了反向最大值并与运动方向相反, 此 时 $x$ 方向上的摩擦力为运动阻力。此外, 当蛇形机 器人关节模块处于波峰或波谷位置时, 中间部分的 几个关节模块在 $y$ 方向上的摩擦力接近零, 而两端 的几个关节模块在 $y$ 方向上则产生了较大的摩 擦力。

图 8 中, 前三个子图为蛇形机器人前六个关节 模块的质心在 $x$ 方向、法向方向的运动速度以及绝 对角随时间的变化关系，后两个子图为关节模块的 质心在 $x$ 方向、法向方向运动速度与绝对角的相图。 同样针对第五个关节模块，当其处于上拐点或下拐 点位置附近, 即 $\theta_{i}= \pm a_{0}$ 附近时, 其法向运动速度达 到了最大值; 当其处于波峰或波谷位置附近, 即 $\theta_{i}=0$ 附近时， $x$ 方向上的运动速度达到了最大值。 此外, 当蛇形机器人关节模块处于波峰或波谷位置 时，中间部分的几个关节模块在法向方向上的速度 接近零, 而两端的几个关节模块产生了较大的法向 运动速度, 即产生了一定的侧滑现象。

根据以上结果可以得出以下结论。

(1) 蜿蜒运动中的推进力来源于处于拐点附近 关节模块的运动, 主要由沿关节模块法向方向上的 较大滑移所产生的摩擦力而形成的推进力; 蛇形机 器人中间部分的关节连杆产生推进力的贡献程度大 于前后两端部分关节连杆的贡献程度。

(2) 蜿蜒运动中的阻力来源于处于波峰和波谷 位置处关节模块的运动, 而这些位置的关节模块在 前进方向上的运动速度较大并且沿模块侧向方向上 的滑移程度较小。

(3) 蛇形机器人的平面蜿蜒运动可以看作是由 处于拐点附近的关节模块通过侧向滑移产生的摩擦 力推动处于波峰和波谷位置处的关节模块向前 运动。

因此, 当生物蛇以很快的速度进行蜿蜒运动时, 蛇的波峰和波谷处会向上尧起与地面脱离, 从而减 小运动过程中的摩擦阻力, 使得运动能量消耗最小, 运动速度更快 ${ }^{[30]}$ 。 


\section{2 不同摩擦系数比下的前向运动速度}

在具有不同摩擦特性的地面上蜿蜒运动时, 蛇 形机器人的前向运动速度有较大差别, 在光滑的地 板上运动时会出现较为明显的滑移现象而无法很好 地向前运动, 甚至只能在原地摆动, 而在表面较为 粗粘的地面上运动时, 蛇形机器人与地面之间的滑 移程度非常微小, 前向运动速度很快。产生这些不 同运动状态的主要原因是蛇形机器人关节连杆和接 触地面之间摩擦力的各向异性程度决定的。

定义法向和切向摩擦系数之间的比值 $\xi$ 来表示 摩擦力的各向异性程度, 用临界摩擦系数比值 $\xi_{0}$ 表 示当前向运动速度为零时所对应的 $\xi$ 值

$$
\begin{gathered}
\xi=\frac{\mu_{\mathrm{n}}}{\mu_{\mathrm{t}}} \\
\xi_{0}=\left.\xi\right|_{v=0}
\end{gathered}
$$

根据第 1.2 节中蛇形机器人的动力学模型, 在 摩擦力模型中设置不同的切向摩擦系数 $\mu_{\mathrm{t}}$ 、摩擦系 数比 $\xi$ 、波形初始角 $a_{0}$ 、波形个数 $K_{n}$ 、从动轮位置 $l_{0}$, 研究蛇形机器人平面蜿蜒运动中前向运动速度 $v$ 与这些量之间的对应关系, 以及蛇形机器人能够 实现向前蜿蜒运动时 $\xi$ 应满足的条件。

设置仿真参数如表 2 所示, 根据蛇形机器人蜿 蜒运动的动力学模型, 利用 Matlab 的 ode 45 求解, 得到运动速度 $v$ 与摩擦系数、波形初始角等参数之 间的对应关系，如图 9 12 所示。

表 2 不同摩擦系数比下的仿真参数设置

\begin{tabular}{ccccc}
\hline 参数 & 条件 1 & 条件 2 & 条件 3 & 条件 4 \\
\hline 切向摩擦系数 $\mu_{\mathrm{t}}$ & $0.05 \sim 0.3$ & 0.05 & 0.05 & 0.05 \\
波形初始角 $a_{0} /\left(^{\circ}\right)$ & 40 & $30 \sim 60$ & 40 & 40 \\
波形个数 $K_{n}$ & 1 & 1 & $1 \sim 2.5$ & 1 \\
从动轮位置 $l_{0} / \mathrm{m}$ & 0.1059 & 0.1059 & 0.1059 & $0 \sim 0.2118$ \\
连杆长度 $l / \mathrm{m}$ & 0.1059 & 0.1059 & 0.1059 & 0.1059 \\
波形周期 $T / \mathrm{s}$ & 10 & 10 & 10 & 10 \\
连杆质量 $m / \mathrm{kg}$ & 0.268 & 0.268 & 0.268 & 0.268 \\
转动惯量 $J /\left(\mathrm{kg} \cdot \mathrm{m}^{2}\right)$ & 0.001 & 0.001 & 0.001 & 0.001 \\
连杆个数 $N$ & 11 & 11 & 11 & 11 \\
\hline
\end{tabular}

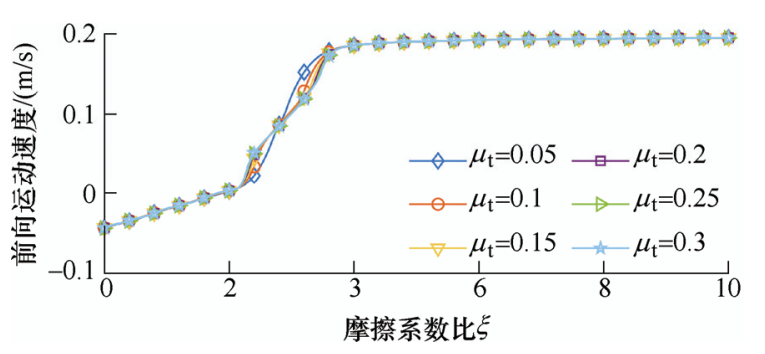

图 9 不同 $\mu_{\mathrm{t}} 、 \xi$ 时的前向运动速度

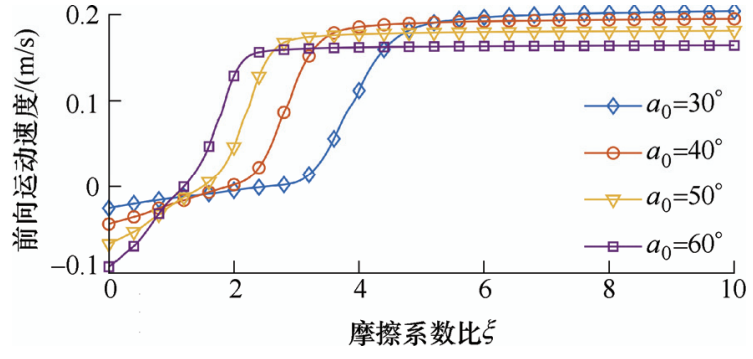

图 10 不同 $a_{0} 、 \xi$ 时的前向运动速度

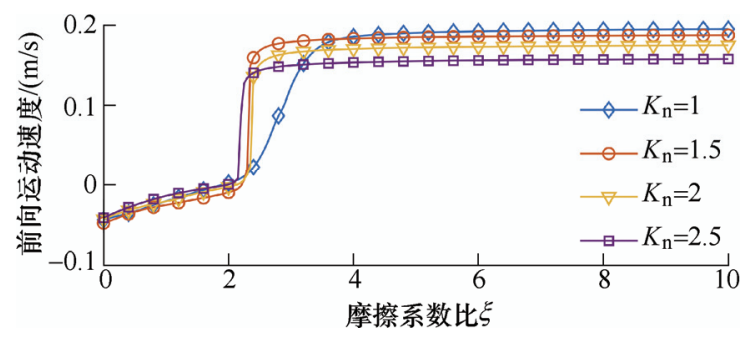

图 11 不同 $K_{n} 、 \xi$ 时的前向运动速度

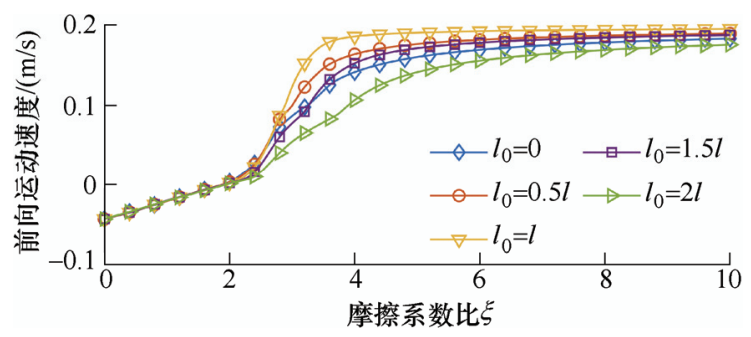

图 12 不同 $l_{0} 、 \xi$ 时的前向运动速度

根据图 9 图 12, 可得如下结论。

(1) 当 $\xi<\xi_{0}$ 时, 蛇形机器人前向运动速度略小 于零, 此时蛇形机器人只能原地打滑, 不能向前 运动。

(2) 当 $\xi>\xi_{0}$ 时, 蛇形机器人开始向前蜿蜒运 动, 运动速度会随着 $\xi$ 的增大而迅速增大并最终趋 于一个极限值，如图 10、图 11 所示，该极限值主 要与波形初始角 $a_{0}$ 、波形个数 $K_{n}$ 有关, 随着 $a_{0} 、 K_{n}$ 的增大而逐渐减小。

(3) 临界摩擦系数比 $\xi_{0}$ 主要与波形初始角 $a_{0}$ 有 关，如图 10 所示， $\xi_{0}$ 随 $a_{0}$ 值的增大而减小，即当 $a_{0}$ 值越大时, 蛇形机器人越容易在更为光滑的地面上 蜿蜒运动 ${ }^{[17,31]}$, 这主要是因为当 $a_{0}$ 值较大时, 蛇形 机器人的关节模块的法向方向和前进运动方向之间 的夹角较小, 因此, 法向摩擦力能够较大程度的抑 制蛇形机器人向后滑移。

(4) 当摩擦系数比 $\xi$ 值相同时, 如图 9 所示, 蛇形机器人的前向运动速度也相同而与摩擦系数的 具体数值无关 ${ }^{[17]}$ 。由式(32)、(33)可以看出, 摩擦力 与摩擦系数之间是线性叠加关系, 当摩擦系数改变 时， $x 、 y$ 方向的摩擦力也改变但两个方向摩擦力 
之间的比值并不变, 因此并不会影响蛇形机器人的 运动速度 ${ }^{[32]}$ 。

(5) 从动轮位置 $l_{0}$ 对蛇形机器人的前向运动速 度 $v$ 有一定程度的影响, 如图 12 所示。摩擦系数比 $\xi$ 在 $2.5 \sim 8$ 的范围内时, 当 $l_{0}=l$ 时, 即从动轮位于 关节连杆的中心位置, 此时前向运动速度最大, 而 在其他位置时前向运动速度都比较小; 当摩擦系数 比足够大时, 从动轮位置对于前向运动速度的影响 程度较小。因此使从动轮安装于关节连杆的中心位 置时能够在一定程度上提高蛇形机器人的前向运动 速度。

综上所述, 蛇形机器人实现平面蜿蜒运动的各 向异性摩擦特性条件应满足蛇形机器人关节模块与 地面之间的法向和切向摩擦系数比值 $\xi$ 大于临界摩 擦系数比值 $\xi_{0}$, 仿真中 $\xi_{0} \approx 3$, 并且当 $\xi>5$ 时, 蛇 形机器人的前向蜿蜒运动速度几乎接近其极限值, 此后通过增大 $\xi$ 的值并不能有效提高蛇形机器人的 前向运动速度。

\section{4 蜿蜒运动的样机实验}

\section{1 蛇形机器人实验样机}

本文采用正交关节结构设计蛇形机器人的实验 样机, 实验样机总长 $2.2 \mathrm{~m}$, 质量 $31.25 \mathrm{~kg}$, 具备 三维运动能力, 如图 13 所示。

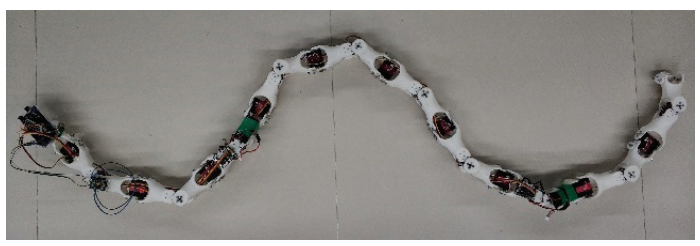

图 13 蛇形机器人实验样机

样机结构主要包括骨架、串行总线舵机、 从动轮、从动轮固定架、控制器、TTL 板、串 行总线及一些紧固零件等。蛇形机器人实验样 机的每个关节均由舵机直接驱动, 舵机之间由 骨架、舵盘连接, 关节结构如图 14 所示, 关节 参数如表 3 所示 ${ }^{[33-34]}$ 。

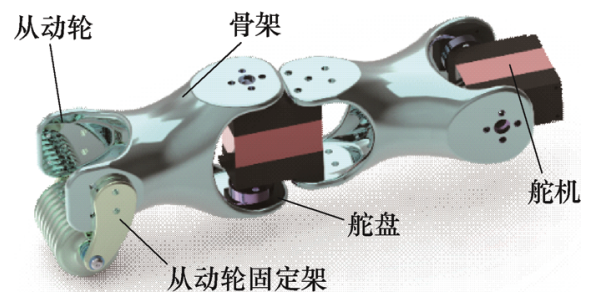

图 14 蛇形机器人正交关节
表 3 蛇形机器人关节参数

\begin{tabular}{cc}
\hline 参数 & 数值 \\
\hline 关节连杆长度 $2 l / \mathrm{m}$ & 0.2118 \\
从动轮位置 $l_{0} / \mathrm{m}$ & 0.079 \\
关节连杆数量 $N$ & 11 \\
关节连杆质量 $m / \mathrm{kg}$ & 0.29 \\
关节连杆传动惯量 $J /\left(\mathrm{kg} \cdot \mathrm{m}^{2}\right)$ & 0.003 \\
偏航角 $\phi /\left(^{\circ}\right)$ & \pm 80 \\
俯仰角 $\psi /\left(^{\circ}\right)$ & \pm 80 \\
离地高度 $h / \mathrm{m}$ & 0.015 \\
\hline
\end{tabular}

除舵机等零件以外, 实验样机中的从动轮用尼 龙 3D 打印成型, 而其他零件则由光敏树脂 3D 打印 成型, 之后直接组装, 避免了一般机加工繁琐的生 产和装配过程。利用 Arduino mega 2560 作为蛇形机 器人的主要控制器, Arduino mega 2560 生成的控制 指令经 UART 串口通过 TTL 板对舵机进行控制, 控 制系统结构如图 15 所示 ${ }^{[34]}$ 。

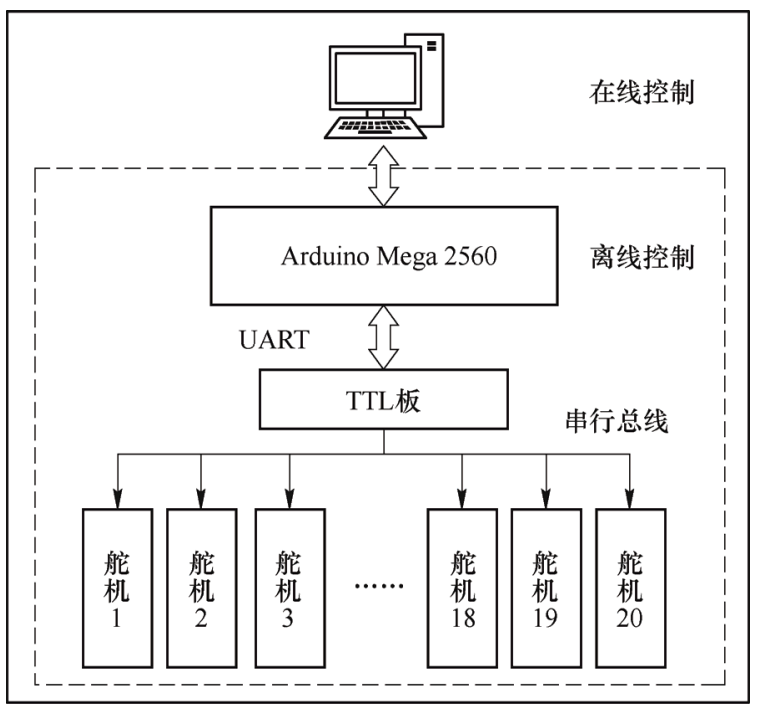

图 15 控制系统结构

\section{2 不同摩擦条件下的实验与仿真比较}

为了测得蛇形机器人在不同摩擦系数比下蜿蜓 运动速度, 需要设置不同的摩擦条件, 根据第 3.2 节中的结论, 前向运动速度与摩擦系数比有关而与 摩擦系数的具体取值无关, 因此, 尽管切向摩擦系 数或法向摩擦系数不一样, 只需要保证不同的摩擦 系数比就可以满足实验所需的不同摩擦条件要 求。根据 2.1 节的结论, 切向摩擦系数由从动轮 转轴的摩擦力矩大小决定, 法向摩擦系数主要由 地面材料和表面性质决定，因此，本文将蛇形机 器人在从动轮固定架的夹紧程度较紧和稍紧两种 情况下在实验室光滑地板上运动, 以及在从动轮 
固定架的夹紧程度正常时在实验室光滑地板、宿 舍走廊地板等四种不同粗䊁程度的地面上运动, 如图 16 所示。

利用测力计分别测得六种情况下的切向摩擦 力和法向摩擦力, 最终得到摩擦系数比值如表 4 所示, 测得蛇形机器人分别在这六种不同摩擦系 数比下取不同波形初始角 $a_{0}$ 、波形个数 $K_{n}$ 时的前 向运动速度, 得到前向运动速度 $v$ 与波形初始角 $a_{0}$ 、 波形个数 $K_{n}$ 、摩擦系数比 $\xi$ 之间的变化规律, 如 图 17〜图 23 所示, 图中实线是实验结果, 虚线是 仿真结果。

表 4 六种情况下的摩擦系数比值

\begin{tabular}{cc}
\hline 环境条件 & 摩擦系数比 $\xi$ \\
\hline 较紧拧紧 & 0.73 \\
稍紧拧紧 & 1.8 \\
实验室光滑地板 & 5.3 \\
宿舍走廊地板 & 8.75 \\
大厅粗徏地板 & 11.25 \\
实验室粗徏地板 & 15.3 \\
\hline
\end{tabular}

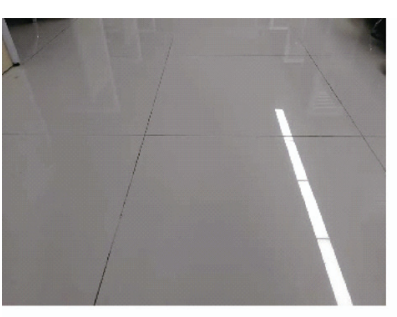

(a) 实验室光滑地板

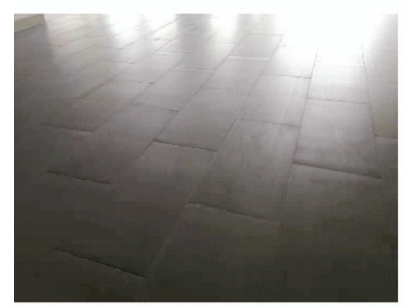

(c) 大厅粗糙地板

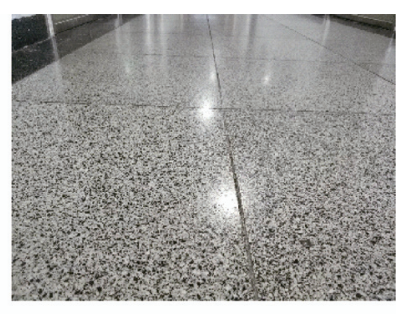

(b) 宿舍走廊地板

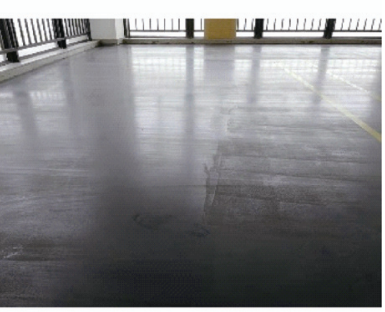

(d) 实验室粗粘地板
图 16 不同粗糙程度的实验地面

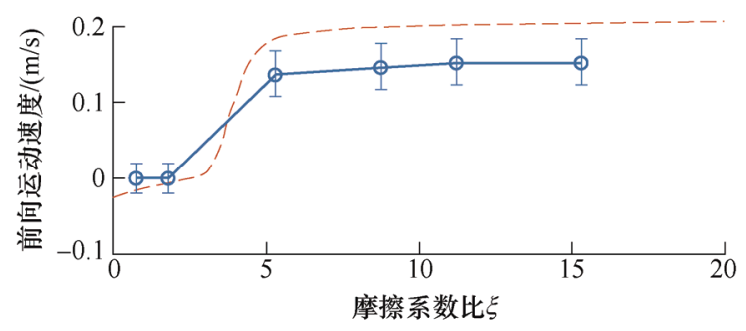

图 $17 a_{0}=30^{\circ}$ 的前向运动速度

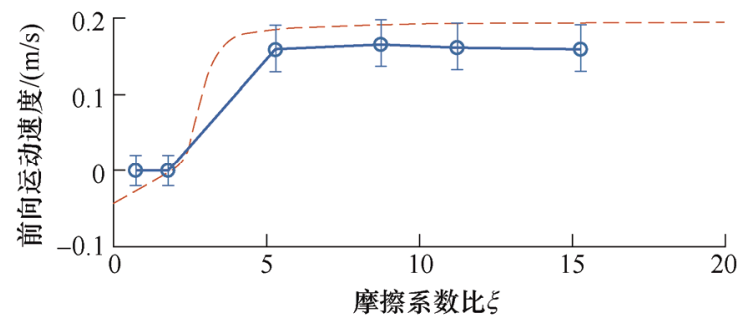

图 $18 a_{0}=40^{\circ}$ 的前向运动速度

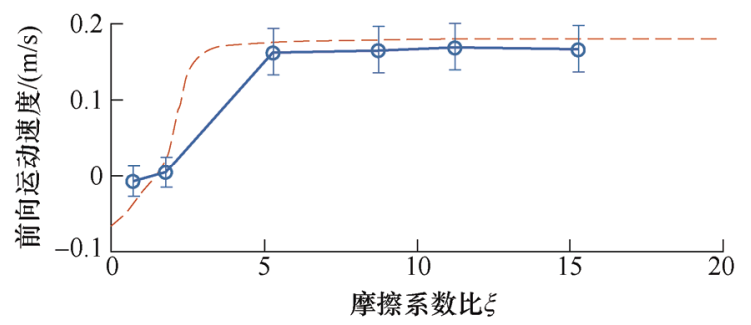

图 $19 a_{0}=50^{\circ}$ 的前向运动速度

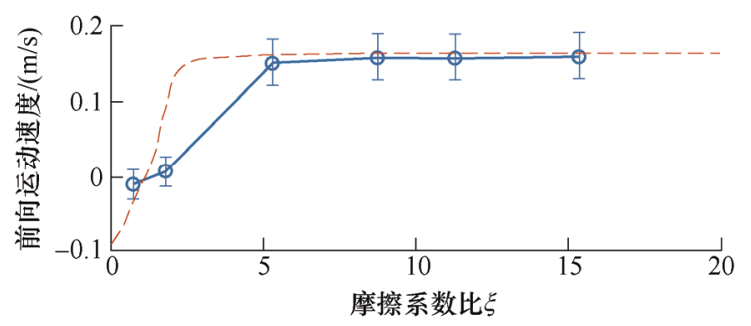

图 $20 a_{0}=60^{\circ}$ 的前向运动速度

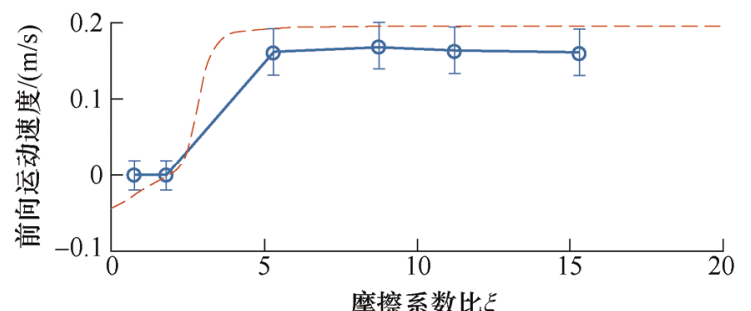

图 $21 K_{n}=1$ 时的前向运动速度

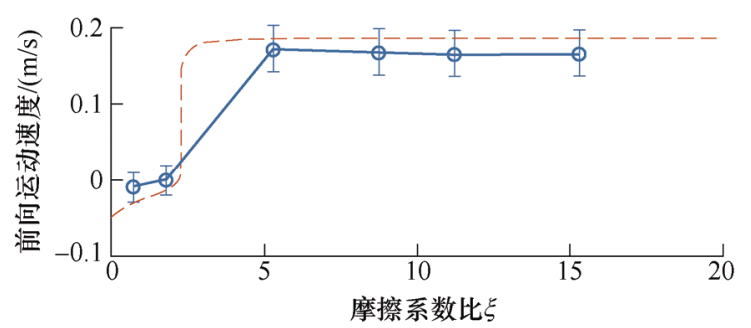

图 $22 K_{n}=1.5$ 时的前向运动速度

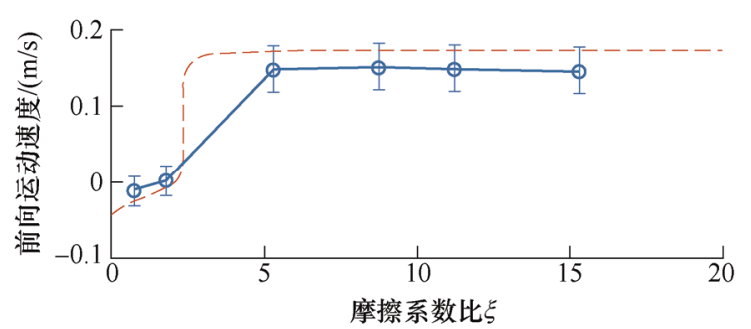

图 $23 K_{n}=2$ 时的前向运动速度 
根据以上实验结果, 可得以下结论。

(1) 在不同摩擦系数比 $\xi$ 下, 实验与理论结果 具有相似的运动规律, 但实验中当前向运动速度为 零时所对应的临界摩擦系数比 $\xi_{0}$ 值比理论值要小 一些, 实验值约为 2 , 这可能是由于实际的蛇形机 器人关节模块与地面之间的粘滞摩擦特性较大, 从 而减小了摩擦力的各向异性程度, 使得实际中蛇形 机器人要比理论中更容易实现蜿蜒运动;

(2) 当摩擦系数比 $\xi$ 比较大时, 实际的蛇形机 器人前向运动速度 $v$ 会随着波形初始角 $a_{0}$ 、波形个 数 $K_{n}$ 的增大, 先增大然后逐渐减小, 当 $a_{0}=50^{\circ}$ 和 $K_{n}=1.5$ 时对应的前向运动速度较大;

(3) 当摩擦系数比 $\xi$ 较大时, 蛇形机器人的前 向运动速度极限值比理论值要小, 这可能是由于实 际的蛇形机器人关节结构存在一定的柔性, 与理论 中所假设的刚性连杆模型有一定的差距。另外, 蛇 形机器人关节的驱动舵机在运动过程中, 由于连接 各个舵机的串行总线之间相互干扰而导致控制信号 产生噪声, 使舵机产生随机抖动的现象, 从而影响 了蛇形机器人的运动速度。

\section{5 结论}

本文针对带从动轮的蛇形机器人, 利用动力学 建模、仿真分析和样机实验相结合的方法, 对蛇形 机器人蜿蜒运动的摩擦学机理和推进条件进行了 研究。

（1）考虑实际结构中从动轮在蛇形机器人关节 连杆上的位置, 在 Liljebäck 和 Pettersen 关于蛇形机 器人平面蜿蜒运动的动力学模型的基础上进行了改 进, 改进后的模型可以描述蛇形机器人从动轮安装 在关节连杆的任何位置的蛇形机器人运动情况, 通 过仿真得到, 当从动轮位于关节连杆中心位置比位 于其他位置时的前向运动速度要大。

(2) 提出一种库伦-粘滞混合摩擦力模型, 通过 将库伦摩擦力模型中的非光滑区域替换为光滑的粘 滞摩擦力模型, 能够有效提高动力学模型的求解效 率与计算稳定性。

(3) 基于所建立的动力学模型与摩擦力模型, 通过对运动过程中蛇形机器人各个关节模块摩擦力 的仿真分析, 解释了蜿蜒运动的摩擦学推进机理: 蛇形机器人蜿蜒运动是由位于波峰和波谷间的关节 模块通过侧向滑移产生的摩擦力推动位于波峰和波 谷位置处的关节模块向前运动, 而运动阻力来源于 位于波峰和波谷位置处的关节模块的运动, 因此,
当把位于波峰和波谷位置处的关节模块与地面脱 离, 能够有效减少运动阻力和能量消耗, 增大蛇形 机器人的运动速度。

（4）基于蜿蜒运动的摩擦推进机理, 对不同摩 擦系数下的摩擦力和前向运动速度进行了仿真, 通 过在不同切向摩擦系数、摩擦系数比、波形初始角、 波形个数、从动轮位置下的仿真, 得到蛇形机器人 前向蜿蜒运动速度变化规律, 前向运动速度与摩擦 系数比有关, 而与切向摩擦系数、法向摩擦系数的 具体取值无关，增大波形初始角度能够增大蛇形机 器人在更为光滑的表面上运动的可能。

(5) 通过带从动轮的蛇形机器人样机实验, 设 置不同的摩擦条件, 对实现蜿蜒运动的摩擦学推进 条件与运动速度进行了实验验证，结果表明在不同 摩擦系数比下, 实验与理论具有相似的运动规律, 并对仿真与实验结果的误差进行了分析, 研究结论 对于实现蛇形机器人的结构优化, 步态规划与运动 控制具有重要的理论意义与实际指导价值。

\section{参 考 文 献}

[1] 李斌, 马书根, 王越超, 等. 一种具有三维运动能力的 蛇形机器人的研究[J]. 机器人, 2004(6): 506-509.

LI Bin, MA Shugen, WANG Yuechao, et al. Development of a snake-like robot with $3 \mathrm{~d}$ mobility[J]. ROBOT, 2004(6): 506-509.

[2] SHAO L, GUO B, WANG Y, et al. An overview on theory and implementation of snake-like robots[C]// 2015 IEEE International Conference on Mechatronics and Automation (ICMA), 2015: 70-75.

[3] LILJEBäCK P, PETTERSEN K Y, STAVDAHL Ø, et al. A review on modelling, implementation, and control of snake robots[J]. Robotics and Autonomous Systems, 2012, 60(1): 29-40.

[4] 陈丽, 王越超, 李斌. 蛇形机器人研究现况与进展 $[\mathrm{J}]$. 机器人, 2002(6): 559-563.

CHEN Li, WANG Yuechao, LI Bin. Present state and future direction towards snake-robot research[J]. ROBOT, 2002(6): 559-563.

[5] 丁建宁, 胡友耀, 杨继昌, 等. 蛇类表皮摩擦学性能的 AFM 研究 $[C] / / 2006$ 全国摩擦学学术会议, 2006: 4.

DING Jianning, HU Youyao, YANG Jichang, et al. Friction properties of snake epidermis scales by afm[C]// 2006 National Tribology Conference, 2006: 4.

[6] 张吴, 戴振东, 杨松祥. 蛇腹鳞的结构特点及其摩擦行 为 [J]. 南京航空航天大学学报, 2008, 40(3): 360-363. 
ZHANG Hao, DAI Zhendong, YANG Songxiang. Structure and friction characteristics of snake abdomen[J]. Journal of Nanjing University of Aeronautics and Astronautics, 2008, 40(3): 360-363.

[7] OWEN T. Biologically inspired robots : Snakelike locomotors and manipulators by shigeo hirose[J]. Robotica, 1994, 12(3): 282-282.

[8] KOMURA H, YAMADA H, HIROSE S. Development of snake-like robot acm-r8 with large and mono-tread wheel[J]. Advanced Robotics, 2015, 29(17): 1081-1094.

[9] 王国彪, 陈殿生, 陈科位, 等. 仿生机器人研究现状与 发展趋势[J]. 机械工程学报, 2015, 51(13): 27-44.

WANG Guobiao, CHEN Diansheng, CHEN Kewei, et al. The current research status and development strategy on biomimetic robot[J]. Journal of Mechanical Engineering, 2015, 51(13): 27-44.

[10] 王生栋. 蛇形机器人结构设计与运动控制研究 [D]. 哈 尔滨工业大学, 2016.

WANG Shengdong. Research on structure design and motion control of snake-like robot[D]. Harbin Institute Of Technology, 2016.

[11] 黄恒, 颜国正, 熊翔. 蛇形机器人的运动策略 [J]. 电机 与控制学报, 2002, 6(3): 249-251.

HUANG Heng, YAN Guozheng, XIONG Xiang. Motion planning for snake-like robot[J]. Electric Machines and Control, 2002, 6(3): 249-251.

[12] 高琴. 基于振荡器的蛇形机器人 CPG 运动控制方法[D]. 大连理工大学, 2017.

GAO Qin. A cpg locomotion control method of a snake robot based on oscillators[D]. Dalian University Of Technology, 2017.

[13] 叶长龙, 马书根, 李斌, 等. 三维蛇形机器人巡视者 II 的开发 [J]. 机械工程学报, 2009, 45(5): 128-133.

YE Changlong, MA Shugen, LI Bin, et al. Development of a three dimensional snake-like robot perambulator II[J]. Journal of Mechanical Engineering, 2009, 45(5): 128-133.

[14] 郁树梅, 王明辉, 马书根, 等. 水陆两栖蛇形机器人的 研制及其陆地和水下步态 [J]. 机械工程学报, 2012, 48(9): $18-25$.

YU Shumei, WANG Minghui, MA Shugen, et al. Development of an amphibious snake-like robot and its gaits on ground and in water[J]. Journal of Mechanical Engineering, 2012, 48(9): 18-25.

[15] BAYSAL Y A, ALTAS I H. Modelling and simulation of a wheel-less snake robot[C]// $2020 \quad 7$ th International Conference on Electrical and Electronics Engineering
(ICEEE), 2020: 285-289.

[16] MA Shugen, TADOKORO N, LI Bin, et al. Analysis of creeping locomotion of a snake robot on a slope[C]// 2003 IEEE International Conference on Robotics and Automation (Cat. No.03CH37422), 2003: 2073-2078.

[17] WU Xiaodong, MA Shugen. Adaptive creeping locomotion of a cpg-controlled snake-like robot to environment change[J]. Autonomous Robots, 2010, 28(3): 283-294.

[18] LU Zhenli, FENG Dayu, XIE Yafei, et al. Study on the motion control of snake-like robots on land and in water[J]. Perspectives in Science, 2016, 7: 101-108.

[19] 张丹凤, 李斌, 王立岩. 基于连续体模型的蛇形机器人 质心速度跟踪控制方法 [J]. 机器人, 2017, 39(6): 829-837.

ZHANG Danfeng, LI Bin, WANG Liyan. Tracking control method of the centre-of-mass velocity for a snake-like robot based on the continuum model [J]. ROBOT, 2017, 39(6): 829-837.

[20] LI Bin, MA Shugen, WANG Yuechao, et al. Environment-adaptable locomotion of a snake-like robot[C]// 2004 IEEE International Conference on Robotics and Biomimetics, 2004: 584-588.

[21] 陈丽, 王越超, 马书根, 等. 蛇形机器人行波运动的研 究[J]. 机械工程学报, 2004, 40(12): 38-43.

CHEN Li, WANG Yuechao, MA Shugen, et al. Research on traveling wave motion of snake-like robot[J]. Journal of Mechanical Engineering, 2004，40(12): 38-43.

[22] MA Shugen, TADOKORO N. Analysis of creeping locomotion of a snake robot on a slope[C]// Robotics and Automation, 2003. Proceedings. ICRA '03. IEEE International Conference on, 2003: 1.

[23] LIM J, YANG Weixin, SHEN Y, et al. Analysis and validation of serpentine locomotion dynamics of a wheeled snake robot moving on varied sloped environments[C]// 2020 IEEE/ASME International Conference on Advanced Intelligent Mechatronics (AIM), 2020: 1069-1074.

[24] BAYSAL Y A, ALTAS I H. Optimally efficient locomotion of snake $\operatorname{robot}[\mathrm{C}] / / 2020$ International Conference on INnovations in Intelligent SysTems and Applications (INISTA), 2020: 1-6.

[25] PETTERSEN K Y, LILJEBÄCK P, STAVDAHL Ø, et al. Snake robots from biology to nonlinear control[J]. IFAC Proceedings Volumes, 2013, 46(23): 110-115.

[26] LILJEBÄCK P, PETTERSEN K Y, Ø S, et al. 
Controllability analysis of planar snake robots influenced by viscous ground friction[C]// 2009 IEEE/RSJ International Conference on Intelligent Robots and Systems, 2009: 3615-3622.

[27] LILJEBÄCK P. Snake robots: Modelling, mechatronics, and control[M]. New York: Springer, 2013.

[28] YIN Hui, CHEN Y H, YU Dejie, et al. Adaptive robust control for a soft robotic snake: A smooth-zone approach[J]. Applied Mathematical Modelling, 2020, 80: 454-471.

[29] 陈祺, 占雄, 徐鉴. 振动驱动移动机器人直线运动的滑 移分岔 [J]. 力学学报, 2016, 48(4): 792-803.

CHEN Qi, ZHAN Xiong, XU Jina. Sliding bifurcations of rectilinear motion of a three-phase vibrarion-driven system subject to coulomb dry friction[J]. Chinese Journal of Theoretical and Applied Mechanics, 2016, 48(4): 792-803.

[30] HIROSE S, YAMADA H. Snake-like robots[J]. IEEE Robotics \& Automation Magazine, 2009, 16(1): 88-98.

[31] WANG Zhelong, GAO Qin, ZHAO Hongyu. Cpg-inspired locomotion control for a snake robot basing on nonlinear oscillators[J]. Journal of Intelligent \& Robotic Systems,
2017, 85(2): 209-227.

[32] 唐超权, 马书根, 李斌, 等. 具有环境适应能力的蛇形 机器人仿生控制方法 $[\mathrm{J}]$. 中国科学: 信息科学, 2014, 44(5): 647-663.

TANG Chaoquan, MA Shugen, LI Bin, et al. Bionic control method of snake-robot with environmental adaptability[J]. SCIENTIA SINICA Informationis, 2014, 44(5): 647-663.

[33] DALU S S, DALU P S. Design and development of modular snake robot and implementation of locomotive gaits[C]// 2019 IEEE Pune Section International Conference (PuneCon), 2019: 1-5.

[34] XUE Yongjiang, ZHANG Ye, ZHENG Wenxiong, et al. Design, control and experiment of a snake-like robot with gripper[C]// 2020 17th International Conference on Ubiquitous Robots (UR), 2020: 50-55.

作者简介: 刘旭鸠, 男, 1996 年出生, 博士研究生。主要研究方向为机 器人动力学与控制。

E-mail: liuxupeng@xs.ustb.edu.cn

郜志英(通信作者), 女, 1979 年出生, 博士, 教授。主要研究方向为机 械系统动力学, 非线性动力学及控制。

E-mail: gaozhiying@me.ustb.edu.cn 